\title{
Effects of lyophilization and storage temperature on Wuchereria bancrofti antigen sensitivity and stability
}

\author{
Edem Y. Agbozo ${ }^{1,2}$, Edward Dumashie $^{2}$, Daniel A. Boakye ${ }^{2}$ and Dziedzom K. de Souza ${ }^{2 *}$
}

\begin{abstract}
Objective: Antigen-based rapid diagnostic tests for Lymphatic filariasis (LF) do not come with external quality control (QC) materials, and research and disease control programmes rely on stored positive samples. This study was undertaken to evaluate the use of lyophilized Wuchereria bancrofti antigen positive plasma samples to serve as QC materials for LF diagnostic tests. 10 well characterized $W$. bancrofti positive samples were lyophilized and stored at 4 , 28 and $40^{\circ} \mathrm{C}$. The samples were evaluated using the Alere Filariasis Test Strips before lyophilization, and after 1 and 3 months of storage. The sensitivity and stability of the lyophilized samples were evaluated.

Results: The results revealed a loss of sensitivity and stability with increasing temperature and duration of storage. The results are further discussed in terms of the use of dried blood spot (DBS) in diagnostic studies on LF, and the need for thoughtful DBS preparation and storage.
\end{abstract}

Keywords: Wuchereria bancrofti antigen, Lyophilization, Dried blood spots, Freeze-drying

\section{Introduction}

Lymphatic filariasis (LF) is found in tropical and subtropical regions of the world, where it is a major public health problem [1]. The availability of tools and strategies for the control of the disease $[2,3]$ led to World Health Assembly resolution (WHA 50.29) calling on member states to work towards the elimination of LF as a public health problem by 2020 [4]. The World Health Organization (WHO) launched the Global Programme to Eliminate Lymphatic Filariasis (GPELF) in 2000, with the principal objective of breaking the cycles of transmission of Wuchereria bancrofti and Brugia spp. through the application of annual mass drug administration (MDA) [5] to entire at-risk populations for a period of 5-7 years. Thus, more than 6.7 billion cumulative treatments were provided to disease endemic communities by 2015 [6]. Impact evaluation assessments are based on the prevalence of infection using $W$. bancrofti antigen rapid

\footnotetext{
*Correspondence: ddesouza@noguchi.ug.edu.gh

${ }^{2}$ Department of Parasitology, Noguchi Memorial Institute for Medical Research, University of Ghana, Accra, Ghana

Full list of author information is available at the end of the article
}

diagnostic tests (RDTs) or microscopy for microfilaria presence [7].

As part of the GPELF, millions of RDTs as either the BinaxNOW immunochromatographic (ICT) cards [8] and the Alere Filariasis Test Strips (FTS) [9] have been and are currently being used in LF endemic countries, being integrated in LF endemicity mapping, MDA monitoring and stopping, and post-MDA surveillance $[7,10]$. The BinaxNOW ICT and Alere FTS detect circulating filarial antigen (CFA) - a $200 \mathrm{kDa}$ W. bancrofti antigen-in human blood [9]. Though the BinaxNOW ICT was the first to be developed, challenges with its short shelf life of 3 months at ambient temperature, cost, narrow time window for reading test results and false-positive rates prompted the need for the development of the improved Alere FTS [9]. The Alere FTS is an in vitro, visually read, immunochromatographic test for the qualitative assessment of $W$. bancrofti antigen in blood, utilizing a colloidal gold-labeled polyclonal antibody (PAb) and a monoclonal antibody (MAb) specific for W. bancrofti. In a positive sample, W. bancrofti antigen combines with the gold-labeled PAb, which is then captured by the MAb forming a pink diagnostic

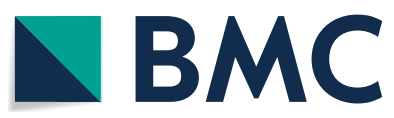

(c) The Author(s) 2018. This article is distributed under the terms of the Creative Commons Attribution 4.0 International License (http://creativecommons.org/licenses/by/4.0/), which permits unrestricted use, distribution, and reproduction in any medium, provided you give appropriate credit to the original author(s) and the source, provide a link to the Creative Commons license, and indicate if changes were made. The Creative Commons Public Domain Dedication waiver (http://creativecommons.org/ publicdomain/zero/1.0/) applies to the data made available in this article, unless otherwise stated. 
line. While the Alere FTS has an in-built internal procedural control, the use of external controls is recommended to ensure the test reagents are working and that the test is correctly performed. Research and LF control programmes therefore rely on stored samples as quality control (QC) materials. These samples are stored either in the form of plasma or serum in fridges/freezers, or dried blood spots (DBS) stored on silica gel and/or in fridges/freezers. This study sought to test the use of lyophilized (freeze-dried) W. bancrofti antigen positive plasma, adapted from a similar method used for HIV rapid tests [11] and malaria [12], as QC material for Alere FTS. Lyophilization was used because it provides a better temperature and humidity-control during the drying process, compared to DBS $[13,14]$.

The samples used for this study are blood samples collected from LF positive individuals, as part of ongoing research activities [15]. Briefly, study participants were tested during the day for W. banrofti antigen using the Alere FTS. Individuals positive for antigen were followed for night blood collection, for the identification of microfilaria. From each participant, $2 \mathrm{ml}$ of night blood was collected (in EDTA coated tubes). $1 \mathrm{ml}$ of blood was analyzed for microfilaria using nucleopore filtration. The remaining blood samples were centrifuged, the plasma separated from the pellets, and frozen at $-80{ }^{\circ} \mathrm{C}$.

\section{Main text}

Methods

Dried W. bancrofti infected blood preparation

For baseline reactivity (6 months after storage), 10 frozen plasma samples with known parasite counts (Table 1) were thawed at room temperature and tested on the Alere FTS following the manufacturer's instructions. From the remaining plasma samples, six (6) aliquots (75 $\mu \mathrm{l}$ each) per sample were lyophilized using a freeze drier (Lyotrap, Ultra Freeze Dryer LF/LYO/04/1, LTE Scientific). Two aliquots from each of the samples were stored in a refrigerator set at $4{ }^{\circ} \mathrm{C}$, at room temperature $\left(28{ }^{\circ} \mathrm{C} \pm 3{ }^{\circ} \mathrm{C}\right)$ and in a dry incubator set at $40{ }^{\circ} \mathrm{C}$, respectively. These were tested after a period of one and 3 months.

\section{Dried blood rehydration and FTS testing}

On the day of testing ( 1 and 3 months after storage), the lyophilized samples were rehydrated with $1 \times$ PBS solution ( $\mathrm{pH}$ 7.3). $75 \mu \mathrm{l}$ of PBS was added to the sample tube, and incubated at room temperature for $1 \mathrm{~h}$. The mixture was gently mixed using a pipette and transferred onto the Alere FTS for reactivity testing.

\section{Reading and scoring of test results}

For all tests undertaken (i.e. baseline, 1 and 3 months) the results were scored as negative (test band absent $=0$ ) or positive (test band present). Even though the Alere FTS is a qualitative tool, the intensity of the test line was assessed visually to provide a semi-quantitative

Table 1 Results of Alere FTS testing at baseline, one and 3 months storage at different temperatures

\begin{tabular}{|c|c|c|c|c|c|c|c|c|}
\hline \multirow[t]{2}{*}{ Samples } & \multirow{2}{*}{$\begin{array}{l}\text { Microfilaria/ml } \\
\text { of blood }\end{array}$} & \multirow[t]{2}{*}{ Baseline testing } & \multicolumn{3}{|c|}{ Results at 1 month } & \multicolumn{3}{|c|}{ Results at 3 months } \\
\hline & & & $4^{\circ} \mathrm{C}$ & $28^{\circ} \mathrm{C}$ & $40^{\circ} \mathrm{C}$ & $4^{\circ} \mathrm{C}$ & $28^{\circ} \mathrm{C}$ & $40^{\circ} \mathrm{C}$ \\
\hline A & 1 & +++ & +++ & + & +++ & +++ & + & ++ \\
\hline B & 1 & +++ & +++ & ++ & ++ & + & - & + \\
\hline$C$ & 6 & + & + & + & + & + & + & + \\
\hline $\mathrm{D}$ & 43 & ++ & + & + & + & + & + & - \\
\hline E & 7 & +++ & ++ & ++ & ++ & ++ & - & + \\
\hline $\mathrm{F}$ & 1 & +++ & ++ & ++ & + & ++ & + & + \\
\hline G & 6 & +++ & ++ & ++ & ++ & + & - & - \\
\hline $\mathrm{H}$ & 1 & ++ & - & - & - & - & - & - \\
\hline I & 57 & +++ & +++ & +++ & ++ & ++ & + & + \\
\hline J & 2 & ++ & +++ & +++ & +++ & + & - & - \\
\hline Number positive & & 10 & 9 & 9 & 9 & 9 & 5 & 6 \\
\hline Sensitivity & & $100 \%$ & $90 \%$ & $90 \%$ & $90 \%$ & $90 \%$ & $50 \%$ & $60 \%$ \\
\hline Mean intensity score & & 2.5 & 2.0 & 1.7 & 1.7 & 1.4 & 0.5 & 0.7 \\
\hline Standard deviation & & 0.707 & 1.054 & 0.949 & 0.949 & 0.843 & 0.527 & 0.675 \\
\hline Standard error of mean & & 0.224 & 0.333 & 0.300 & 0.300 & 0.267 & 0.167 & 0.213 \\
\hline p values & & & 0.188 & 0.055 & 0.055 & 0.008 & 0.004 & 0.004 \\
\hline
\end{tabular}

$\mathrm{p}$ values represent the comparison of means to the baseline 
score as previously described [16]. Thus, positive results were scored on a scale of $1-3 ; 1(+)$ being a test band being lighter in intensity than the control band, $2(++)$ being a test band having a similar intensity as the control band, and $3(+++)$ being a test band with a brighter intensity than the control band. The control band has to be positive for any test to be valid. A false negative test was defined as a sample identified as positive at baseline (before lyophilization) and appearing as negative after lyophilization. All readings were undertaken and scored by two individuals.

\section{Data analysis}

The baseline test results before lyophilization were used as the reference standard, in estimating the sensitivity of using the lyophilized samples. The sensitivity was estimated by combining the results for each temperature storage condition and expressed as:

$$
\begin{aligned}
& \text { Sensitivity } \\
& =\frac{\text { Total samples positive under each storage condition }}{\text { Total samples positive at baseline }} \\
& \quad \times 100
\end{aligned}
$$

From the semi-quantitative scoring, the mean intensity scores for the baseline, storage temperature and period of storage were computed and test of significance between results were undertaken using the Wilcoxon matched pairs rank test at $95 \%$ confidence interval. Graphs and statistical analyses were undertaken using GraphPad Prism version 7.0 (GraphPad Software, Inc., La Jolla, CA).

\section{Results}

\section{Sensitivity of W. bancrofti lyophilized samples}

The results of the evaluations are presented in Table 1. One sample lost total reactivity after lyophilization, while five others were false negative at 28 and $40{ }^{\circ} \mathrm{C}$ after 3 months. From the results, $90 \%$ sensitivity was observed when the lyophilized samples were tested after 1 month. After 3 months of storage, the sensitivity of the lyophilized samples stored at $4{ }^{\circ} \mathrm{C}$ remained unchanged, while that of the samples stored at 28 and $40{ }^{\circ} \mathrm{C}$ decreased to 50 and $60 \%$ respectively.

\section{Assessing W. bancrofti antigen stability}

The results showed a decrease in antigen stability with increasing temperature and storage duration (Fig. 1). Compared to the baseline, the results indicate a slight but insignificant loss $(\mathrm{p}=0.188)$ in stability in the lyophilized samples stored at $4{ }^{\circ} \mathrm{C}$ for 1 month. However, storage at $4{ }^{\circ} \mathrm{C}$ for 3 months resulted in further significant loss in

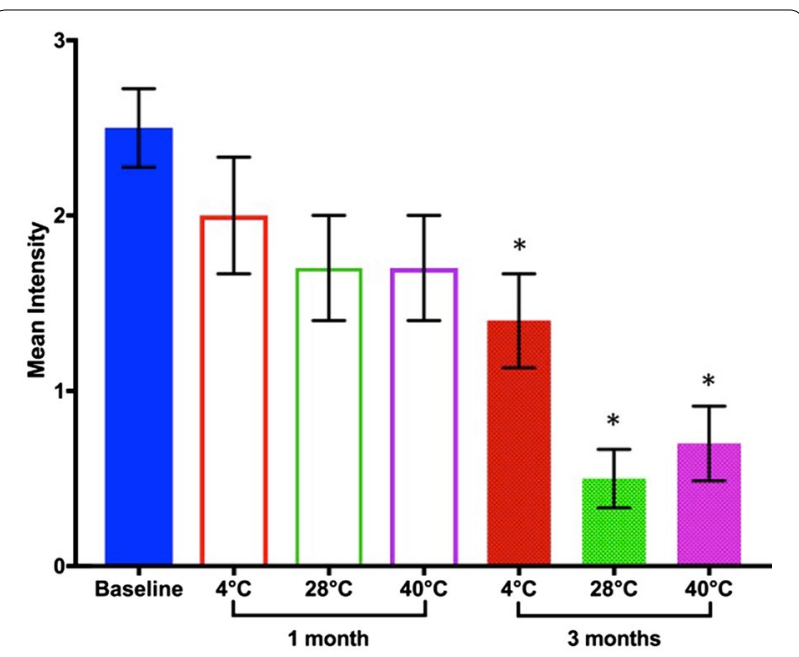

Fig. 1 Change in antigen stability with temperature and storage time. Error bars represent the standard errors of the mean. ${ }^{*}$ denotes significance compared to baseline $(p<0.01)$

stability $(\mathrm{p}=0.008)$ compared to the baseline, and no differences compared to the 1 month storage $(\mathrm{p}=0.125)$.

Samples stored at $28{ }^{\circ} \mathrm{C}$ for a month showed an insignificant loss $(\mathrm{p}=0.055)$ in stability compared to the baseline. Storage at 3 months resulted in a much significant loss compared to the baseline $(\mathrm{p}=0.004)$ and the 1 -month storage $(\mathrm{p}=0.031)$.

Storing samples at $40{ }^{\circ} \mathrm{C}$ revealed an insignificant loss in stability $(\mathrm{p}=0.055)$ at 1 month, compared to the baseline results. However, storage at 3 months led to a significant loss in stability compared to the baseline $(\mathrm{p}=0.004)$ and the 1 -month storage $(\mathrm{p}=0.016)$.

The results indicate that higher temperatures affect the stability of the W. bancrofti antigen. Further, long-term storage at higher temperatures results in further loss in antigen stability, with implications for quantitative experiments relying on the use of DBS. Studies evaluating the relative performance of plasma and DBS from W. bancrofti positive blood samples showed lower positivity and sensitivity for DBS compared to plasma [17], even though both plasma and DBS were stored at the same conditions of $-20{ }^{\circ} \mathrm{C}$ (short term) and $-80{ }^{\circ} \mathrm{C}$ (long term). In their study, the only difference in temperature conditions was during the drying stage of the DBS and storage of DBS (in sealed plastic containers) at $4{ }^{\circ} \mathrm{C}$ or in hand luggage during transportation. Their studies also showed a higher mean antigen concentration in plasma compared to DBS. Other studies also showed lower positivity and correlations for DBS compared to serum $[18,19]$, though the storage conditions of the DBS in these studies was not mentioned. Of course, the results from these studies could be explained by the fact that less serum (and 
therefore antigen) is available in the DBS. However, the effects of temperature on further reducing the stability of the antigen in the DBS, as a result of denaturation, must not be ignored.

Our study therefore provides evidence of the effects of drying and temperature on W. bancrofti antigen stability. Studies have shown the effect of temperature on lysosomal enzyme activity during the preparation and storage of DBS [20]. In HIV RDT testing for example, DBS stored at 37 and $45{ }^{\circ} \mathrm{C}$ were shown to have good stability until 8 weeks, but when stored at $50{ }^{\circ} \mathrm{C}$ it showed good stability until week four. The study concluded that in areas with high temperatures DBS can be stored at room temperature and tested within 4 weeks [21]. In many cases DBS are further stored with desiccants, leading to a further 5\% moisture loss during storage [22].

Proteins offer opportunities in disease treatment and diagnostics. However, in order to maintain their properties, proteins need to be stabilized against physical and chemical degradation [23]. Lyophilization improves protein-storage stability, ease of shipping and transportation by removing water [24]. While it has the advantages of; being a low-temperature process with less thermodenaturation risk, controlling moisture level and enabling better stability of proteins, it also has the disadvantage of inducing conformational instability as a result of the freezing and drying process [13, 14]. Important characteristics of lyophilized products include long-term stability, short reconstitution time and maintenance of the characteristics of the original products. However, lyophilization is not a process that can easily be carried out in the field, unlike the DBS. It requires venous blood collection (compared to DBS from finger-prick blood), cold storage, a lyophilizer, and as such is more time consuming and expensive. Nonetheless for QC purposes, we consider it superior to storage through DBS, during which, it is challenging to control the moisture content coupled with varying environmental (drying) temperatures-under which LF studies are undertaken-which may result in denaturation and loss of protein/antigen activity. In this study, temperatures of $28^{\circ} \mathrm{C}\left( \pm 3^{\circ} \mathrm{C}\right)$ and $40{ }^{\circ} \mathrm{C}$ were used as these represent the range of temperatures in LF endemic regions in Ghana and possible temperatures under which DBS may be stored in the absence of a fridge/freezer.

Currently, the Alere FTS kits are not supplied with positive control materials. Until W. bancrofti recombinant positive control antigens become commercially available, research and national control programmes will continue to rely on stored samples (where available) as QC materials. Given the progress made through GPELF and the low/limited numbers of positive individuals being detected, sample storage methods should carefully be considered if these are to serve as QC for future studies and programme evaluation activities, most especially for studies relying on quantitative assessment methods and the determination of positive cut-off thresholds. While the use of dried parasitized blood has been evaluated as QC materials for HIV [11] and malaria [12] tests, and may be applicable to LF, further developments of the method aimed at stabilizing the antigen [25], followed by field testing may enhance the utility of lyophilized W. bancrofti antigen-positive blood samples in the future.

In conclusion, the results from this study would suggest that dried W. bancrofti antigen-positive blood samples stored for long periods above $4{ }^{\circ} \mathrm{C}$ should carefully be used in quantitative experiments, given the loss in sensitivity over time. While the DBS is a simpler and cheaper method than collecting samples in EDTA tubes, care must be taken in the storage of these samples in order to obtain the best possible results from their use.

\section{Limitations}

The main challenge to this study was the mall number of samples evaluated. This is due to challenges in obtaining large numbers of well-characterized samples at the current stage of the GPELF in Ghana. While other samples positive with $W$. bancrofti day blood antigen are available, sample size was limited to 10 in order to avoid using all samples at the expense for future positive control materials. The sample volumes available also prevented the preparation of DBS for comparison. Another challenge to the FTS reading may be the subjectivity of the readings by the testing personnel, especially for very faint test bands. However, having two personnel assess the results helped resolve this challenge.

\section{Abbreviations \\ DBS: dry blood spot; EDTA: ethylene diamine tetraacetic acid; FTS: filaria test strip; GPELF: Global Programme for the Elimination of Lymphatic Filariasis; HIV: human immunodeficiency virus; LF: Iymphatic filariasis; MDA: mass drug administration; NMIMR: Noguchi Memorial Institute for Medical Research; PBS: phosphate buffer saline; QC: quality control; RDT: rapid diagnostic test; WHO: World Health Organization.}

\section{Authors' contributions}

DKdS and DAB conceived the study. EYA, ED and DKdS designed the experiments. EYA and ED performed the experiments. EYA and DKdS analyzed the data. EYA, ED, DAB and DKdS wrote the paper. All authors read and approved the final manuscript.

\section{Author details \\ ${ }^{1}$ Accra Technical University, Accra, Ghana. ${ }^{2}$ Department of Parasitology, Noguchi Memorial Institute for Medical Research, University of Ghana, Accra, Ghana.}

\section{Acknowledgements}

We are grateful to Prof. Regina Appiah-Opong for freeze-drying the samples for us. 


\section{Competing interests}

The authors declare that they have no competing interests.

\section{Availability of data and materials}

All data generated and analyzed during this study are included in this published article.

\section{Consent to publish}

Not applicable.

\section{Ethical approval and consent to participate}

The study received approval from the ethics committee of the Ghana Health Service (GHS-ERC: 04112/2016). It was also reviewed by the NMIMR IRB (CPN 062/16-17) with Federal Wide Assurance Registration (FWA 00001824). Written informed consent was received from all study participants.

\section{Funding}

This project was undertaken as part of the EDCTP2 programme supported by the European Union, through Grant Number 98595 to DKdS. The funding source has no role in the design of the study, in the collection, analysis and interpretation of the data, or in the publication of the study results.

\section{Publisher's Note}

Springer Nature remains neutral with regard to jurisdictional claims in published maps and institutional affiliations.

Received: 11 June 2018 Accepted: 7 July 2018

Published online: 11 July 2018

\section{References}

1. WHO. Global programme to eliminate lymphatic filariasis: progress report, 2016. Wkly Epidemiol Rec. 2017;92:589-608.

2. Ottesen EA. Lymphatic filariasis: treatment, control and elimination. Adv Parasitol. 2006:61:395-441.

3. Ottesen EA, Duke BO, Karam M, Behbehani K. Strategies and tools for the control/elimination of lymphatic filariasis. Bull World Health Organ. 1997;75(6):491-503.

4. WHO. Monitoring and epidemiological assessment of mass drug administration in the global programme to eliminate lymphatic filariasis: a manual for national elimination programmes. WHO/HTM/NTD/PCT/2011, vol. 4. Geneva: WHO; 2011. p. 1-79.

5. WHO. Guideline - alternative mass drug administration regimens to eliminate lymphatic filariasis. WHO/HTM/NTD/PCT/2017, vol. 4. Geneva: WHO; 2017

6. Molyneux DH, Savioli L, Engels D. Neglected tropical diseases: progress towards addressing the chronic pandemic. Lancet. 2017;389(10066):312-25.

7. WHO. Monitoring and epidemiological assessment of mass drug administration in the global programme to eliminate lymphatic filariasis: a manual for national elimination programmes. In: WHO, editor. WHO/ HTM/NTD/PCT/2011, vol. 4. Geneva:WHO; 2011. p. 1-79.

8. Weil GJ, Lammie PJ, Weiss N. The ICT filariasis test: a rapid-format antigen test for diagnosis of bancroftian filariasis. Parasitol Today. 1997;13(10):401-4.

9. Weil GJ, Curtis KC, Fakoli L, Fischer K, Gankpala L, Lammie PJ, et al. Laboratory and field evaluation of a new rapid test for detecting Wuchereria bancrofti antigen in human blood. Am J Trop Med Hyg. 2013;89(1):11-5.
10. WHO. Lymphatic filariasis: a handbook for national elimination programmes, vol. WHO/HTM/NTD/PCT/2013.10. Geneva: World Health Organization; 2013.

11. Parekh BS, Anyanwu J, Patel H, Downer M, Kalou M, Gichimu C, et al. Dried tube specimens: a simple and cost-effective method for preparation of HIV proficiency testing panels and quality control materials for use in resource-limited settings. J Virol Methods. 2010;163(2):295-300.

12. Aidoo M, Patel JC, Barnwell JW. Dried Plasmodium falciparum-infected samples as positive controls for malaria rapid diagnostic tests. Malar J. 2012;11:239.

13. Chang LL, Pikal MJ. Mechanisms of protein stabilization in the solid state. J Pharm Sci. 2009;98(9):2886-908.

14. Kasper JC, Friess W. The freezing step in lyophilization: physico-chemical fundamentals, freezing methods and consequences on process performance and quality attributes of biopharmaceuticals. Eur J Pharm Biopharm. 2011;78(2):248-63.

15. de Souza DK, Ahorlu CS, Adu-Amankwah S, Otchere J, Mensah SK, Larb IA, et al. Community-based trial of annual versus biannual single-dose ivermectin plus albendazole against Wuchereria bancrofti infection in human and mosquito populations: study protocol for a cluster randomised controlled trial. Trials. 2017;18(1):448.

16. Chesnais CB, Missamou F, Pion SDS, Bopda J, Louya F, Majewski AC, et al. Semi-quantitative scoring of an immunochromatographic test for circulating filarial antigen. Am J Trop Med Hyg. 2013;89(5):916-8.

17. Masson J, Douglass J, Roineau M, Aye K, Htwe K, Warner J, et al. Relative performance and predictive values of plasma and dried blood spots with filter paper sampling techniques and dilutions of the lymphatic filariasis Og4C3 antigen ELISA for samples from Myanmar. Trop Med Infect Dis. 2017;2(2):7.

18. Itoh M, Gunawardena NK, Qiu XG, Weerasooriya MV, Kimura E. The use of whole blood absorbed on filter paper to detect Wuchereria bancrofti circulating antigen. Trans R Soc Trop Med Hyg. 1998;92(5):513-5.

19. Lalitha P, Ravichandran M, Suba S, Kaliraj P, Narayanan RB, Jayaraman K. Quantitative assessment of circulating antigens in human lymphatic filariasis: a field evaluation of monoclonal antibody-based ELISA using blood collected on filter strips. Trop Med Int Health. 1998;3(1):41-5.

20. Supriya M, De T, Christopher R. Effect of temperature on lysosomal enzyme activity during preparation and storage of dried blood spots. J Clin Lab Anal. 2018;32(1):e22220.

21. Yassin M, Eldaif WA, Elkhider IM. Evaluation of dry blood spot (DBS) stored at different temperature for detection of HIV antibodies by using different rapid tests. Surgery. 2013:2:S2-12.

22. Denniff P, Spooner N. Effect of storage conditions on the weight and appearance of dried blood spot samples on various cellulose-based substrates. Bioanalysis. 2010;2(11):1817-22.

23. Carpenter JF, Pikal MJ, Chang BS, Randolph TW. Rational design of stable lyophilized protein formulations: some practical advice. Pharm Res. 1997;14(8):969-75.

24. Wang W. Lyophilization and development of solid protein pharmaceuticals. Int J Pharm. 2000;203(1-2):1-60.

25. Garzon-Rodriguez W, Koval RL, Chongprasert S, Krishnan S, Randolph TW, Warne NW, et al. Optimizing storage stability of lyophilized recombinant human interleukin-11 with disaccharide/hydroxyethyl starch mixtures. J Pharm Sci. 2004;93(3):684-96.

Ready to submit your research? Choose BMC and benefit from

- fast, convenient online submission

- thorough peer review by experienced researchers in your field

- rapid publication on acceptance

- support for research data, including large and complex data types

- gold Open Access which fosters wider collaboration and increased citations

- maximum visibility for your research: over 100M website views per year

At BMC, research is always in progress.

Learn more biomedcentral.com/submissions 